\title{
A Post-Processing Deconvolution Step for Wavelet-Based Image Denoising Methods
}

\author{
Max Mignotte
}

\begin{abstract}
In this letter, we show that the performance of image denoising algorithms using wavelet transforms can be improved by a post-processing deconvolution step that takes into account the inherent blur function created by the considered wavelet based denoising system. The interest of the proposed deblurring procedure is illustrated on denoised images reconstructed by shrinkage of curvelet and undecimated wavelet coefficients. Experimental results reported here show that the proposed post-processing technique yields improvements in term of image quality and lower mean square error, especially when the image is corrupted by strong additive white Gaussian noise.
\end{abstract}

Index Terms-Curvelet, deblurring, deconvolution, image denoising, nonnegative Garrote shrinkage, undecimated wavelet transform.

\section{INTRODUCTION}

$\mathbf{O}$ VER the last decade, there has been considerable interest in wavelet-based denoising methods [1]-[5]. In these schemes, the basic idea consists in projecting the noisy image onto a properly selected orthogonal set of basis functions and, before reconstruction, in applying a thresholding rule that will tend to remove more noise (mostly represented by low wavelet coefficients) than important image edge information (mostly represented by high value coefficients).

Amongst the numerous existing wavelet transforms (WT) [6], the most promising decomposition techniques, in the context of image denoising, seem to be the undecimated wavelet (UDW) [7] and the curvelet [5] transforms. Besides, one of the most efficient rule for the above-mentioned thresholding step is the so-called nonnegative Garrote shrinkage which offers several advantages over both hard and soft shrinkages [8].

In order to further improve the denoising results, many researchers have then proposed and studied (sometimes very sophisticated) ideas of variation on this, initially simple, basic denoising procedure. To this end, some of these techniques exploit level-dependent or spatially adaptive threshold functions based (for example) on the correlation or the a priori knowledge of the statistical distribution of the wavelet coefficients to name a few.

In this letter, we will show that the performance of the basic wavelet based image denoising procedures can be improved by a simple post-processing iterative deconvolution step, taking into

Manuscript received August 31, 2006; revised January 30, 2007. The associate editor coordinating the review of this manuscript and approving it for publication was Dr. Konstantinos N. Plataniotis.

The author is with the Département d'Informatique et de Recherche Opérationnelle (DIRO), Université de Montréal, Montréal, QC H3C 3J7, Canada (e-mail: mignotte@iro.umontreal.ca).

Color versions of one or more of the figures in this paper are available online at http://ieeexplore.ieee.org.

Digital Object Identifier 10.1109/LSP.2007.896183 account the inherent blur function created by the sparsity constraint induced by the considered wavelet shrinkage operation. The interest of the proposed procedure is herein illustrated on denoised images reconstructed from the nonnegative Garrote shrinkage rule of curvelet and UDW coefficients. Experimental results reported here shows that this method yields improvements in term of image quality and lower mean square error (mse), especially when the image is corrupted by strong additive white Gaussian (AWG) noise.

\section{Proposed ApPROACH}

Let us consider that the transformation from $x$ (the unblurred original image to be recovered) to $x_{\text {den }}$ (the observed blurred version after the considered wavelet-based denoising algorithm) is described by the following degradation model,

$$
x_{\text {den }}=h * x+n_{r}
$$

where $n_{r}$ is the remaining corrupting additive noise, $h *$ represents the nonlinear, nonstationary (i.e., spatially variant) blur operator generated by the considered wavelet based denoising process. In this model, this operator is clearly nonlinear (the [WT-THRESHOLDING-INVERSE WT] ${ }^{1}$ operation on a sum of images is not the sum of [WT-THRESHOLDING-INVERSEWT] images). Besides, as already mentioned, this operator is also inherently spatially variant since the thresholding operation is dependant of the 2-D signal repartition. These properties make very difficult any deconvolution and/or point spread function (PSF) estimation method. By assuming that the operation " $h *$ " can be estimated, a simple method would consist in defining the deconvolution problem as the search of the global minima of the following energy function

$$
E(x)=\left\|x_{\mathrm{den}}-h * x\right\|^{2}
$$

which can be easily solved with a simple steepest descent procedure which moves the estimate iteratively in the negative gradient direction, i.e., $\hat{x}^{[n+1]}=\hat{x}^{[n]}-\gamma \nabla E(x)$ or equivalently with the so-called Landweber iteration [9]

$$
\hat{x}^{[n+1]}=\hat{x}^{[n]}+\gamma\left(h^{\#} *\left(x_{\mathrm{den}}-h * \hat{x}^{[n]}\right)\right)
$$

where $n>0, \hat{x}^{[0]}=x_{\text {den }}, \gamma$ is the step size of the iterative gradient descent and $h^{\#}(i, j)=h(-i,-j)$ (the coordinates $(i, j)$ denoting the discrete pixel locations).

1i.e., the basic denoising method using a thresholding in the wavelet domain. 
Although the nonlinear and nonstationary blur generated by the (Garrote) shrinkage operation on the wavelet coefficients (before image reconstruction) is difficult to parametrically estimate, the blur function " $h *$ " nevertheless remains known and can be numerically simulated. More precisely, the blur degradation generated by a wavelet denoising system on a 2-D signal is simply the [WT-THRESHOLDING-INVERSEWT] operation (w.r.t. the considered wavelet transform). In this way, let $\Upsilon()=.h *$ be the [WT-THRESHOLDING-INVERSEWT] operator, the Landweber iteration given by (3) can now be written

$$
\hat{x}^{[n+1]}=\hat{x}^{[n]}+\gamma \cdot \Upsilon\left(x_{\text {den }}-\Upsilon\left(\hat{x}^{[n]}\right)\right)
$$

where $\Upsilon^{\#}=h^{\#} *=\Upsilon$ since the generated blur degradation is the same for an inverted image (also herein assumed to be toroidal) and the hard constraint that each pixel value of $\hat{x}^{[n+1]}$ $(\forall n)$ is in the interval $[0,255]$ can also be efficiently added at each iteration of this procedure.

Since the $\Upsilon()=$. [WT-THRESHOLDING-INVERSEWT] operation is dependant of the scale of the input signal, better results will thus be obtained if we rescale, at each iteration, the residual signal $\left(x_{\text {den }}-\Upsilon\left(x^{[n]}\right)\right)$ w.r.t. the scale of $\Upsilon\left(x^{[n]}\right)$.

In this iterative unconstrained deconvolution approach, in which the residual image provides the update direction of the local gradient descent, $x_{\text {den }}$ is sufficiently close to the optimal undegraded solution (because $x_{\text {den }}$ is already widely denoised) to ensure the uniqueness and the reliability of the final deblurred estimate. Nevertheless, one can also impose a smoothness constraint to this iterative procedure in order to make both the optimization problem well posed and to find a better solution. Herein, we have also used, a sparsity constraint by simply alternating between the Landweber iteration given by (4) and a wavelet based denoising step (this regularization strategy was also proposed in [10]). ${ }^{2}$ To this end, we have used the considered wavelet-based and shrinking operation given by $\Upsilon($.$) with a low threshold value (smaller that the one used in$ the initial denoising process since the image is already widely denoised in this deblurring step).

\section{EXPERIMENTAL RESULTS}

The performance of the proposed deconvolution procedure is herein illustrated on denoised images provided by the nonnegative Garrote shrinkage operation of curvelet and UDW coefficients. In all the experiments, we have considered the gradient-descent iterative procedure defined in (4) with $\gamma=0.1$ and in which we impose, at each iteration, the rescaling of the residual signal and the hard constraint that each pixel value is in the interval $[0,255]$. The convergence criterion of the iterative procedure is given by checking if the residual image is lower than a given threshold, namely, we use $\left|x_{\text {den }}-\Upsilon\left(x^{[n]}\right)\right|<$ $(\sigma / 2.5)$ (combined with a minimum of three iterations).

\footnotetext{
${ }^{2}$ This regularization strategy makes well posed the optimization deconvolution process by a priori promoting an acceptable deblurred image solution having property of sparse frequential representation (in the wavelet domain w.r.t the considered curvelet or UDW transform). This regularization strategy, already used in Bayesian restoration/deconvolution and tomographic reconstruction approaches, is equivalent to the use of a complexity prior [17] whose goal is to penalize estimates with high complexity in a data-compression sense.
}

For the UDW-based denoised images, we constrain the Landweber procedure by using, every each $\lfloor\sigma / 5\rfloor$ iteration, a wavelet-based denoising step with a threshold value $t=2.5$ and one scale level. For the curvelet transform, we use the unconstrained iterative procedure since we have experimentally found that the mse results are not sensitively improved by the sparsity constraint. The overall algorithm is summarized in pseudocode in Table I.

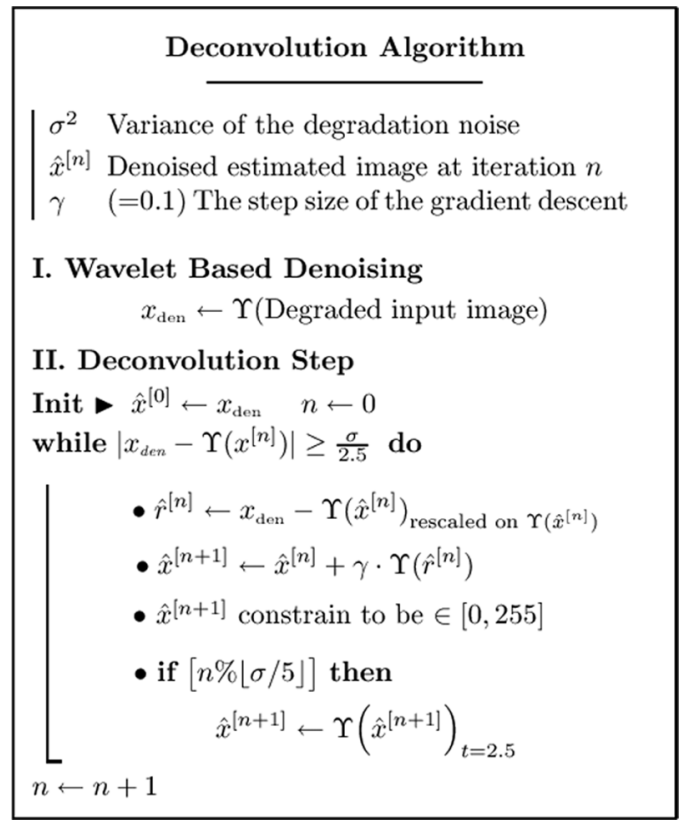

Algo 1: Deconvolution algorithm for wavelet-based image denoising methods.

\section{A. WT Parameters}

We have chosen parameters ensuring competitive denoising results for each degradation models.

- For the curvelet transform, we have used the CurveLab toolbox implementing the (wrapping 2.0.1. version) Fast Discrete Curvelet Transform in C++ (written by Lexing Ying) and available on-line at http://www.curvelet.org/. We use, as parameters of this curvelet transform, five scales (including the coarsest nondirectional wavelet scale), 12 angles in the second coarsest scale (and a curvelet is used at the finest scale).

- For the UDW transform, we use four scales.

For the shrinkage operation, we have used the nonnegative Garrote shrinkage function with (in order to ensure competitive denoising results) $t=\sigma$ for the curvelet transform and $t=$ $\{12,17,23,28,36\}$ for the UDW transform and for respectively a AWG noise degradation model with $\sigma=\{10,15,20,25,30\}$.

\section{B. Nonnegative Garrote Shrinkage}

The nonlinear thresholding rule proposed in [17] and also called the amplitude scale invariant Bayes estimator (ABE) rule (related to the "nonnegative Garrote" function suggested by Gao [8]) and which will be used in the UDW transform is

$$
\lambda^{\mathrm{GAR}}=\frac{\left(w^{2}-3 t^{2}\right)_{+}}{w}
$$


TABLE I

Mse Results for Several Denoising Methods. Left: WaVelet (Redundant) Transforms. Middle: Non WaVelet-Based Denoising Methods. Right: Proposed Method With ReSPectively the UDW and CuRVELET BASEd DECONVOLUTION STEP

\begin{tabular}{|c|c|c|c|c|c|c|c|c|c|c|c|c|}
\hline & [1] & [2] & [3] & [4] & [11] & [12] & [13] & {$[14]$} & {$[15]$} & {$[16]$} & $\begin{array}{c}\text { CURV } \\
\text { Deconv. step }\end{array}$ & $\begin{array}{c}\text { UDW } \\
\text { Deconv. step }\end{array}$ \\
\hline \multicolumn{13}{|l|}{$\begin{array}{c}\text { LENA } \\
\sigma / \mathrm{MSE} \\
\end{array}$} \\
\hline $10 / 100$ & 17.87 & - & 19.16 & 19.20 & 18.75 & - & - & 20.94 & 18.45 & 17.91 & $22.71 \rightarrow \mathbf{2 1 . 1 5}$ & $25.20 \rightarrow \mathbf{2 2 . 1 2}$ \\
\hline $15 / 225$ & 26.49 & 29.90 & 28.15 & 28.10 & 28.06 & 25.89 & - & 30.84 & 27.74 & - & $33.69 \rightarrow \mathbf{3 1 . 3 2}$ & $39.11 \rightarrow \mathbf{3 4 . 1 3}$ \\
\hline $20 / 400$ & 35.24 & 40.20 & 37.71 & 37.10 & 37.68 & 34.52 & - & 41.12 & 37.59 & - & $45,19 \rightarrow \mathbf{4 2 . 0 0}$ & $53.98 \rightarrow \mathbf{4 5 . 3 9}$ \\
\hline $25 / 625$ & 44.06 & 50.80 & 47.48 & 46.50 & 47.54 & 43.36 & - & 51.65 & 47.98 & 44.57 & $57.10 \rightarrow \mathbf{5 3 . 1 1}$ & $69.45 \rightarrow \mathbf{5 7 . 5 1}$ \\
\hline $30 / 900$ & - & - & 57.87 & - & $\mathbf{5 7 . 5 5}$ & - & - & 62.96 & - & - & $69.39 \rightarrow \mathbf{6 4 . 3 5}$ & $84.60 \rightarrow \mathbf{6 8 . 1 4}$ \\
\hline \multicolumn{13}{|l|}{$\begin{array}{l}\text { BOATS } \\
\sigma / \mathrm{MSE} \\
\end{array}$} \\
\hline $10 / 100$ & 28.51 & - & 32.10 & - & 28.51 & - & 21.33 & 31.12 & 28.12 & 29.45 & $37.15 \rightarrow \mathbf{3 3 . 8 2}$ & $36.31 \rightarrow \mathbf{3 2 . 5 0}$ \\
\hline $15 / 225$ & 43.96 & - & 47.92 & - & 44.37 & 42.47 & 36.82 & 44.88 & 43.66 & - & $56.64 \rightarrow \mathbf{5 1 . 7 6}$ & $55.69 \rightarrow \mathbf{4 9 . 4 4}$ \\
\hline $20 / 400$ & 59.58 & - & 64.33 & - & 60.26 & 57.42 & 54.33 & 60.96 & 59.85 & - & $76.35 \rightarrow \mathbf{6 9 . 8 1}$ & $77.32 \rightarrow 66.89$ \\
\hline $25 / 625$ & 75.17 & - & 81.37 & - & 76.93 & 72.62 & - & 78.72 & 76.75 & 78.90 & $96.33 \rightarrow \mathbf{8 8 . 3 4}$ & $98.57 \rightarrow \mathbf{8 5 . 5 1}$ \\
\hline $30 / 900$ & - & - & 96.72 & - & 93.77 & - & 100.48 & 99.79 & - & - & $116.60 \rightarrow \mathbf{1 0 7 . 2 0}$ & $123.30 \rightarrow \mathbf{1 0 3 . 1 7}$ \\
\hline
\end{tabular}

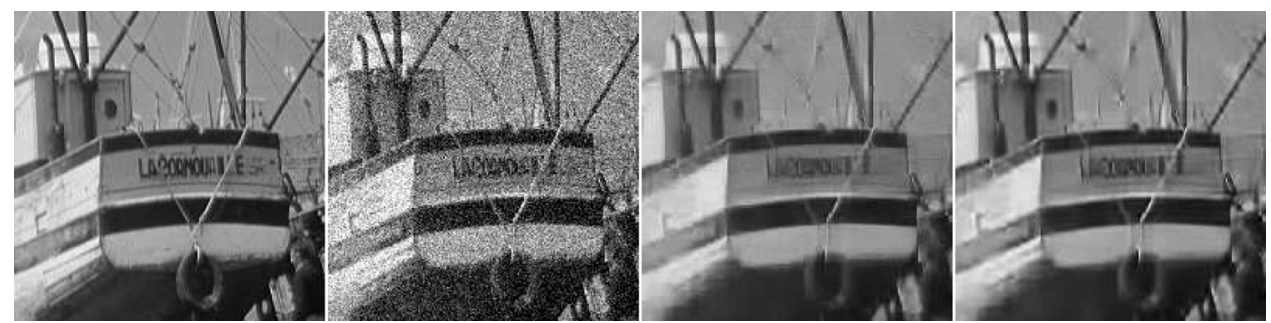

Fig. 1. Left to right: original cropped subregion of the BOATS image, noisy images corrupted with a Gaussian noise $(\sigma=30)$, obtained denoising result with UDW (mse $=123.30)$, and obtained result after our deconvolution procedure initialized with the previous image $(\mathrm{mse}=103.17)(\mathrm{cf}$. Table I).

where $(.)_{+}$denotes the positive part operator, defined as $(x)_{+}=$ $\max \{x, 0\}, t$ is the threshold, $w$ and $\lambda^{\mathrm{GAR}}$ represent respectively the wavelet coefficient before and after the nonnegative Garrote thresholding rule.

Because the curvelet transform is complex and not norm-preserving (and therefore the variance of the noisy curvelet coefficients will depend on its scale and orientation [5]), we have used the generalized nonnegative Garrote shrinkage function to complex values proposed in [18] with the individual variance $\tilde{\sigma}_{w}^{2}$ computed, in our application (in order to save computational time) from the norm of each individual curvelet, as proposed by Lexing Ying (and giving similar results compared to Monte-Carlo simulations [5]) and a scale dependant value for $k$ [5], namely $k=1.5$ for the first scale and $k=1$ for the others, with

$$
\lambda^{\mathrm{GAR}}=w\left(1-\frac{k t \tilde{\sigma}_{w}}{|w|^{2}}\right)_{+}
$$

where $|w|$ is the modulus of the complex wavelet coefficient $w$ and $t$ is the threshold.

\section{Comparisons and Discussion}

We now present a set of experimental results and comparisons illustrating the performance of the proposed deblurring approach. For the experiments, we have replicated the degradation models used in the evaluation of state-of-the-art methods described in [1]-[4] for the best existing wavelet-based methods and [11]-[16] for the best state-of-the-art non-wavelet-based methods. In these experiments, original images are LENA and BOATS (of size $512 \times 512$ ) and the variance of the Gaussian noise vary from 100 to 900 . We have summarized the mse results in Table I. Let us note that the peak signal-to-noise ratio (PSNR) is sometimes given by some authors and is herein converted in Table I in mse measures by mse $=\left(255 \times 10^{-(\mathrm{PSNR} / 20)}\right)^{2}$. The best mse results provided by the existing denoising algorithm and the results provided by our approach for each degradation level are indicated in bold. For our approach, the first mse number is the mse result obtained before the deblurring step and the second (at the right of the arrow) is the mse result after our deblurring step.

Fig. 1 shows a denoising restorations result on a cropped (to $200 \times 200$ ) subregion of the BOATS image corrupted with a AWG noise $(\sigma=30)$, before and after our deconvolution procedure. We can notice that the proposed method is more efficient for denoising image corrupted with strong AWG noise (and for which the denoised image is particularly blurred by the wavelet-based denoising system), especially for the UDWbased denoising technique for which mse are improved about 15\%-20\% (compared to the basic denoising procedure) (see Table I) thus allowing to obtain some competitive denoising results for the BOATS image. We have included in Fig. 2 a crosssection of the original BOATS image along with its noisy version and the initial wavelet denoised estimate, and finally, the reconstructed image with the proposed deconvolution scheme in order to visually see the improvements generated by our deblurring step. We have also combined the two wavelets denoising 


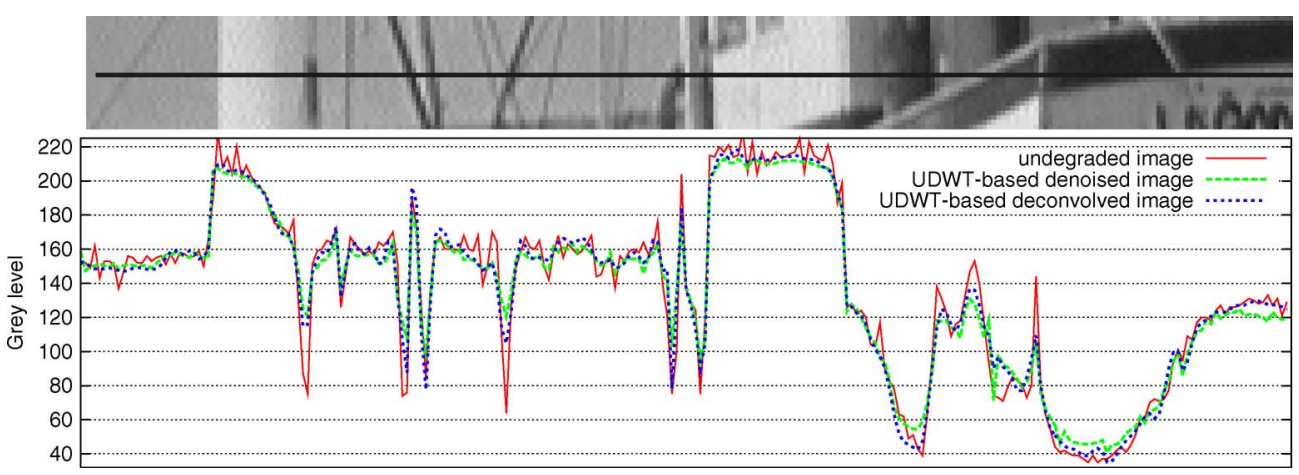

Fig. 2. Cross section of the 208th line (from column 128 to 380) of the original BOATS image (solid line) and the initial UDW denoised estimate $x_{\text {den }}$ (mse $=$ 123.30 for $\sigma=30$ ) and reconstructed estimate with the proposed deconvolution scheme (mse $=103.17$ ).

TABLE II

MSE Results OBtained With THE PRoposed UDW AND CuRVELET DECONVOLUTION PROCEDURE CONSTRAINED RESPECTIVELY BY THE CURVELET AND UDW SPARSITY CONSTRAINT

\begin{tabular}{||c||c|c||}
\hline \multicolumn{1}{||c||}{} & $\begin{array}{c}\text { CURV }_{2} \\
\text { Deconv. step }\end{array}$ & $\begin{array}{c}\text { UDW }_{2} \\
\text { Deconv. step }\end{array}$ \\
\hline $\begin{array}{c}\text { LENA } \\
\sigma / \text { MSE }\end{array}$ & \multicolumn{2}{||}{} \\
\hline $10 / 100$ & $22.71 \rightarrow \mathbf{2 0 . 4 3}$ & $25.20 \rightarrow \mathbf{2 0 . 9 4}$ \\
\hline $15 / 225$ & $33.69 \rightarrow \mathbf{2 9 . 5 8}$ & $39.11 \rightarrow \mathbf{3 1 . 1 5}$ \\
\hline $20 / 400$ & $45,19 \rightarrow \mathbf{3 9 . 4 0}$ & $53.98 \rightarrow \mathbf{4 1 . 7 4}$ \\
\hline $25 / 625$ & $57.10 \rightarrow \mathbf{4 9 . 5 9}$ & $69.45 \rightarrow \mathbf{5 2 . 7 1}$ \\
\hline $30 / 900$ & $69.39 \rightarrow \mathbf{6 0 . 0 4}$ & $84.60 \rightarrow \mathbf{6 3 . 9 9}$ \\
\hline BOATS & \multicolumn{2}{|c||}{} \\
$\sigma /$ MSE & \multicolumn{3}{|c||}{} \\
\hline \hline $10 / 100$ & $37.15 \rightarrow \mathbf{3 2 . 5 1}$ & $36.31 \rightarrow \mathbf{3 1 . 8 1}$ \\
\hline $15 / 225$ & $56.64 \rightarrow \mathbf{4 9 . 0 2}$ & $55.69 \rightarrow \mathbf{4 6 . 7 2}$ \\
\hline $20 / 400$ & $76.35 \rightarrow \mathbf{6 6 . 2 9}$ & $77.32 \rightarrow \mathbf{6 3 . 1 0}$ \\
\hline $25 / 625$ & $96.33 \rightarrow \mathbf{8 4 . 1 3}$ & $98.57 \rightarrow \mathbf{8 0 . 1 4}$ \\
\hline $30 / 900$ & $116.60 \rightarrow \mathbf{1 0 2 . 5 2}$ & $123.30 \rightarrow \mathbf{9 9 . 3 1}$ \\
\hline \hline
\end{tabular}

methods by constraining the UDW-based deconvolution procedure with the curvelet sparsity constraint and inversely. The obtained mse results are summarized in Table II and indicates that this strategy allows to improve the mse results about $10 \%-25 \%$ (compared to the basic denoising procedure).

The proposed deconvolution procedure takes approximatively between three to ten iterations to converge (each iteration requiring two $\Upsilon($.) operations). The deblurring procedure takes between 15 and 60 s for an AMD Athlon 64 Processor 3500+, $2.2 \mathrm{GHz}, 4435.67$ bogomips, and running on Linux.

\section{CONCLUSION}

In this letter, we have presented a deblurring step for improving the performance of wavelet based image denoising techniques. The interest of the proposed procedure is herein illustrated on denoised images reconstructed from the nonnegative Garrote shrinkage on curvelet and UDW coefficients. The method is simple to implement (considering that a WT is already available) and allows to improve about $10 \%-20 \%$ (compared to the basic wavelet-based denoising procedure) the mse restoration result of image corrupted with strong AWG noise.

\section{REFERENCES}

[1] J. Portilla, V. Strela, M. J. Wainwright, and E. P. Simoncelli, "Image denoising using scale mixtures of Gaussians in the wavelet domain," IEEE Trans. Image Process., vol. 12, no. 11, pp. 1338-1351, Nov. 2003.

[2] S. G. Chang, B. Yu, and M. Vetterli, "Spatially adaptive wavelet thresholding with context modeling for image denoising," IEEE Trans. Image Process., vol. 9, no. 9, pp. 1522-1531, Sep. 2000.

[3] L. Sendur and I. W. Selesnick, "Bivariate shrinkage with local variance estimation,” IEEE Signal Process. Lett., vol. 9, no. 12, pp. 438-441, Dec. 2002.

[4] D. Marpe, H. L. Cycon, G. Zander, and K.-U. Barthel, "Context-based denoising of images using iterative wavelet thresholding," in Proc. SPIE, Visual Communications and Image Processing, C.-C. Jay Kuo, Ed., San Jose, CA, 2003, vol. 4671, pp. 907-914.

[5] J.-L. Starck, E. J. Candes, and D. L. Donoho, "The curvelet transform for image denoising," IEEE Trans. Image Process., vol. 11, no. 6, pp. 670-684, Jun. 2002.

[6] L. Duval, Wits: Where is the Starlet? [Online]. Available: http://lcd. siva.free.fr/siva-wits-where-is-the-starlet.html.

[7] G. Beylkin, "On the representation of operators in bases of compactly supported wavelets," SIAM J. Numer. Anal., vol. 29, pp. 1716-1740, Dec. 1992.

[8] H. Gao, "Wavelet shrinkage denoising using the nonnegative Garrote," J. Comp. Graph. Statist., vol. 7, pp. 469-488, 1998.

[9] L. Landweber, "An iterative formula for fredholm integral equations of the first kind," Amer. J. Math., vol. 73, pp. 615-624, 1951.

[10] M. A. T. Figueiredo and R. D. Nowak, "An EM algorithm for waveletbased image restoration," IEEE Trans. Image Process., vol. 12, no. 8, pp. 906-916, Aug. 2003.

[11] O. G. Guleryuz, "Weighted overcomplete denoising," in Proc. Asilomar Conf. Signals and Systems, Pacific Grove, CA, 2003.

[12] A. Foi, K. Dabov, V. Katkovnik, and K. Egiazarian, "Shape-adaptive DCT for denoising and image reconstruction," in Proc. SPIE Electronic Imaging 2006, Image Processing: Algorithms and Systems V, Jan. 2006, vol. 6064A-18.

[13] T. Rabie, "Robust estimation approach for blind denoising," IEEE Trans. Image Process., vol. 14, no. 11, pp. 1755-1765, Nov. 2005.

[14] L. Shen, M. Papadakis, I. Kakadiaris, I. Konstantinidis, D. Kouri, and D. Hoffman, "Image denoising using a tight frame," IEEE Trans. Image Process., vol. 15, no. 5, pp. 1254-1263, May 2006.

[15] M. Elad and M. Aharon, "Image denoising via sparse and redundant representations over learned dictionaries," IEEE Trans. Image Process., vol. 15 , no. 12 , pp. 3736-3745, Dec. 2006.

[16] K. Hirakawa and T. W. Parks, "Image denoising using total least squares," IEEE Trans. Image Process., vol. 15, no. 9, pp. 2730-2742, Sep. 2006.

[17] M. A. Figueiredo and R. D. Nowak, "Wavelet-based image estimation: An empirical Bayes approach using Jeffreys's noninformative prior," IEEE Trans. Image Process., vol. 10, no. 9, pp. 1322-1331, Sep. 2001.

[18] S. Sardy, "Minimax threshold for denoising complex signals with waveshrink," IEEE Trans. Signal Process., vol. 48, no. 4, pp. 1023-1028, Apr. 2000. 